\begin{abstract}
Sex-biased demography, including sex-biased survival or migration, can impact allele frequency changes across the genome. In particular, we can expect different patterns of genetic variation on autosomes and sex chromosomes due to sex-specific differences in life histories, as well as differences in effective population size, transmission modes, and the strength and mode of selection. Here, we demonstrate the role that sex differences in life history played in shaping short-term evolutionary dynamics across the genome. We used a 25-year pedigree and genomic dataset from a long-studied population of Florida Scrub-Jays (Aphelocoma coerulescens) to directly characterize the relative roles of sex-biased demography and inheritance in shaping genome-wide allele frequency trajectories. We used gene dropping simulations to estimate individual genetic contributions to future generations and to model drift and immigration on the known pedigree. We quantified differential expected genetic contributions of males and females over time, showing the impact of sex-biased dispersal in a monogamous system. Due to female-biased dispersal, more autosomal variation is introduced by female immigrants. However, due to male-biased transmission, more $\mathrm{Z}$ variation is introduced by male immigrants. Finally, we partitioned the proportion of variance in allele frequency change through time due to male and female contributions. Overall, most allele frequency change is due to variance in survival and births. Males and females have similar contributions to autosomal allele frequency change, but males have higher contributions to allele frequency change on the $Z$ chromosome. Our work shows the importance of understanding sex-specific demographic processes in accounting for genome-wide allele frequency change in wild populations.
\end{abstract}

KEYWORDS population genetics | pedigrees | sex chromosomes | sex-biased demography

\title{
Allele frequency dynamics under sex-biased demography and sex-specific inheritance in a pedigreed population
}

\author{
Rose M.H. Driscoll ${ }^{a, 1}$, Felix E.G. Beaudry ${ }^{a, 1,2}$, Elissa J. Cosgrove ${ }^{b}$, Reed Bowman ${ }^{c}$, John W. Fitzpatrick ${ }^{d}$, Stephan J. Schoech ${ }^{\mathrm{e}}$ and $^{\mathrm{s}}$ \\ Department of Biology, University of Rochester, Rochester, NY 14627, b Department of Molecular Biology \& Genetics, Cornell University, Ithaca, NY 14850 , \\ Avian Ecology Program, Archbold Biological Station, Venus, FL 33960, d Cornell Lab of Ornithology, Cornell University, Ithaca, NY 14850 , e Department of \\ Biological Sciences, University of Memphis, Memphis, TN 38152, ${ }^{1}$ Co-first author, ${ }^{2}$ Corresponding authors
}

\section{Introduction}

A fundamental goal of evolutionary biology is to determine the roles of different evolutionary processes in governing allele frequency change over time. The realization that evolution can occur on ecological time scales has generated increased interest in characterizing the drivers of allele frequency change over short timescales in natural populations [1-4]. Furthermore, understanding evolution over contemporary timescales is especially important given its relevance to current issues such as public health [5], conservation policy [6, 7], and agricultural practices [8]. To date, most population genetic studies use allele frequencies estimated from present-day samples to make inferences about the evolutionary processes that generated the observed patterns of genetic variation $[9,10]$, though studies that directly track allele frequencies over time are becoming more common (e.g., [11,12]). Temporal genomic data coupled with knowledge of the population pedigree, or the relationships among all individuals in a population over time, can provide precise estimates of the mechanisms underlying short-term allele frequency dynamics [13].

Genome-wide, allele frequency change is driven by differential survival, reproduction, and dispersal among individuals. In other words, depending on their life history, different individuals have different genetic contributions to a population over time. The expected genetic contribution of an individual is defined as the expected number of alleles inherited from that individual present in the population in future generations, and is determined by both the number of descendants of an individual and the randomness of Mendelian segregation [14, 15]. Individual expected genetic contributions are expected to stabilize after a few generations and can be used to estimate individual reproductive values $[10,14,15]$.

One important factor impacting an individual's genetic contribution over time is its sex, as there are often sex differences in life history. In sexually-reproducing organisms, the expected genetic contributions of males and females should be equal on average [10]. However, different sexes often have different life history traits: depending on their mating system, individuals of different sexes can differ in the variance in number of offspring (i.e., reproductive success $[16,17])$, dispersal likelihood and distance [18], or niche occupation [19]. These differences in life history traits can lead to population-level effects such as a biased adult sex ratio or sex-biased migration rates [20-22]. Differences in life history between individuals of different sexes may impact expected genetic contributions and therefore allele frequency change. Indeed, including sex increases the accuracy of modelling demographic changes [23, 24] and will likely help the 
characterization of sources of allele frequency change over time.

Individual expected genetic contributions can vary across the genome. For example, sex chromosomes should have different patterns of expected genetic contributions compared to autosomes. The two most common sex chromosome systems include $X$ and $Y$ chromosomes, where females are $X X$ and males $X Y(e . g .$, in mammals, beetles, and many dipterans), and Z and W chromosomes, where females are ZW and males ZZ (e.g., in birds, lepidopterans, and strawberries). As a result of sex-specific differences in transmission (inheritance rules) and ploidy (e.g., hemizygosity), expected genetic contributions on sex chromosomes are disproportionately influenced by sex-biased demography. The $\mathrm{X}$ and $\mathrm{Z}$ chromosomes can have different effective population sizes than autosomes and are expected to experience more drift than the autosomes $[25,26]$. Indeed, different patterns of genetic variation on autosomes and sex chromosomes are expected to reflect sex-specific differences in effective population size and transmission modes, as well as in life histories [21, 26-29].

Based on differences in effective population size between autosomes and sex chromosomes, two expectations arise [30-32]. First, males are expected to contribute $\frac{2}{3}$ of $Z$ variation, while females are expected to contribute $\frac{1}{3}$, based on the number of chromosomes in each sex [33]. Second, the ratio of $Z$ to autosome diversity should equal $\frac{3}{4}$ [26]. Departures from these expectations can result from sex-biased demographic processes, fuelling the use of neutral genetic diversity ratios between autosomes and sex chromosomes to infer sex-biased demography over long timescales, (i.e., » $\mathrm{Ne}$ generations). For example, the strength of sexual selection, defined as sex-biased variance in reproductive success [34], can be estimated from genetic diversity differences between $X$ and autosomes [25, 35-37]. Similarly, sex-biased migration can be inferred from sex chromosome versus autosome neutral genetic diversity ratios [27, 38]. These endeavours, however, have important caveats. First, it is challenging (if not impossible) to disentangle which sex-biased processes are contributing to biased genetic diversity ratios when several processes occur jointly, especially in populations with overlapping generations of variable sizes [25]. Second, for inferences of sex-biased migration, timescale has been shown to have important impacts on genetic diversity ratios in humans [33, 39], but timescale is rarely considered in inferences of gene flow [40]. These caveats suggest that approaches complementary to neutral genetic diversity data are necessary to deepen our understanding of the impacts of sex-biased demography in natural populations.

One approach for studying sex-biased demography is using individual genetic contributions. Expected genetic contributions can be estimated from population pedigrees using analytical calculations [41,42] or simulations on the pedigree (gene dropping; [43]). With the advent of high-throughput molecular sequencing, gene dropping has been adopted to simulate changes in allele frequency based on known genotypes. This method has been used to estimate the frequency of known recessive lethals in cattle [44], humans [45] and Soay sheep [46], as well as to identify alleles thought to be under positive selection in Florida Scrub-Jays [13], Soay Sheep [47] and domestic foxes [48]. Population pedigrees are therefore an invaluable resource for studying the impacts of sex-biased demography on allele frequency change over time.

A recent study characterized short-term allele frequency dynamics at autosomal loci in a population of Florida Scrub-Jays (Aphelocoma coerulescens) by combining simulations of expected genetic contributions with genomic data [13]. Florida Scrub-Jays are cooperatively breeding birds with a balanced sex ratio of breeding adults, as these jays pair monogamously [49]. Natal dispersal is limited and female-biased [50-52]. Pairs are socially and genetically monogamous ([53]). Previous work has found that immigration plays a large role in allele frequency change even in a large population of jays [13,54]. A population of jays at Archbold Biological Station (hereafter Archbold) has been closely monitored for over 50 years, resulting in a detailed population pedigree with over 6,000 individuals. This study population provides an unrivalled opportunity to study individual-level effects on short-term allele frequency change.

Here, we extended the work of Chen et al. [13] by developing and applying gene dropping methods for sex chromosomes to directly evaluate the contributions of sex-biased demographic processes and sex-specific inheritance to changes in allele frequencies over time in the Florida Scrub-Jay. We first estimated the expected genetic contributions of individuals, contrasting the role of males and females as well as of immigrants grouped by sex. Using separate models to track different modes of inheritance, we compared the expected genetic contributions of males and females at autosomal and Z-linked loci. Next, to identify loci likely to be under selection, we incorporated genotype information and simulated changes in allele frequency over time given the pedigree. Finally, we partitioned the variance in allele frequency change between years across sexes and demographic groups, and quantified the contributions of males and females and different evolutionary processes to allele frequency change at autosomal and Z-linked loci.

\section{Methods}

\section{Study population and pedigree}

A population of Florida Scrub-Jays at Archbold in Venus, Florida, USA, has been intensively monitored for multiple decades, the northern half since 1969 ([49]) and the southern half since 1989 ([55, 56]). Each bird in this population has been uniquely banded, making immigrants easy to identify. Immigrants are defined as birds born outside the study 
population, but the source population of any given immigrant is not known. The entire population is surveyed every few months to provide detailed records of individual survival. Family groups are closely monitored to assess reproductive success, with new nestlings officially banded at 11 days of age. As a result of these detailed records, we have a fairly comprehensive pedigree of the entire population. To avoid the complications associated with study tract expansion in the 1980s, we truncated the pedigree at 1990, resulting in a pedigree with 6,936 individuals in total by the end of our study period in 2013. We note that there was a minor study tract expansion in the southern end of our field site in 1993 that partially contributed to the observed peak in immigration in 1994 (as we cannot distinguish true immigrants from previously-unbanded residents in the new study tract area); however, we also observed an increase in known immigrants in the northern half of our field site (a consistently monitored area) in 1994. The addition of these territories did not impact overall immigration dynamics. Fieldwork at Archbold Biological Station was approved by the Institutional Animal Care and Use Committees at Cornell University (IACUC 2010-0015), the University of Memphis (0067), the University of Rochester (102153), and Archbold Biological Station (AUP-006-R), and was permitted by the U.S. Fish and Wildlife Service (TE824723-8, TE-117769), the U.S. Geological Survey (banding permits: 07732, 23098), and the Florida Fish and Wildlife Conservation Commission (LSSC-10-00205).

Since 1999, birds in the Archbold population have been sexed using molecular markers, following the protocol of Fridolfsson and Ellegren [57]. A previous study generated genotype data for 3,984 individuals using a custom Illumina iSelect Beadchip containing 15,416 SNPs [54]. These individuals were from a set of 68 territories that were consistently monitored since 1990, and included near-exhaustive sampling in 1989-1991, 1995, and 1999-2013. Here, we used data from a set of 10,731 autosomal SNPs (previously used in [13]; see [13, 54] for more information on filtering and quality control) along with 249 Z-linked and 19 pseudoautosomal SNPs with minor allele frequency $>0.05$.

\section{Expected genetic contributions of males and females}

We begin by calculating the genealogical contribution of each individual, defined as the proportion of a given birth cohort that is descended from that individual. In contrast, the expected genetic contribution of an individual is the expected proportion of alleles in a given birth cohort inherited identical-by-descent from our focal individual. We estimated expected individual genetic contributions as the expected proportion of alleles in a nestling cohort that came from a specific (focal) individual. Expected individual genetic contributions depend on transmission patterns and therefore should to differ for autosomal versus sex-linked loci.

For a given autosomal locus, fathers and mothers each transmit one of their two alleles randomly to each offspring. Thus, the expected genetic contribution of an individual may be obtained by tracing the transmission of their alleles through the pedigree. The expected genetic contribution of an individual at an autosomal locus $\left(G_{\text {auto }}\right)$ is:

$$
G_{\text {auto }}=\frac{1}{n} \sum_{m} \sum_{p}\left(\frac{1}{2}\right)^{g p}
$$

where $n$ is the number of nestlings in the birth cohort, $m$ is the number of nestlings in the cohort related to the focal individual (their descendants), $p$ is the number of paths in the pedigree linking the focal individual and a given descendant, and $g_{p}$ is the number of generations between the focal individual and the descendant along a given path.

For a given $\mathrm{Z}$ chromosome locus, fathers transmit one of their two $\mathrm{Z}$ chromosomes randomly to each offspring, but mothers invariably transmit their single $Z$ chromosome to their male offspring only. The estimated genetic contribution of an individual at a $Z$ chromosome locus in a given year $\left(G_{Z}\right)$ is:

$$
G_{Z}=\frac{1}{n} \sum_{m} \sum_{p}\left(\frac{1}{2}\right)^{h_{p}}\left(\frac{1}{2}\right)^{j_{p}}(1)^{k_{p}}(0)^{l_{p}}
$$

where $n, m$, and $p$ are as described above. $h_{p}$ represents the number of male-to-male (i.e., father-to-son) transmission events, $j_{p}$ represents the number of male-to-female (i.e., father-to-daughter) transmission events, $k_{p}$ represents the number of female-to-male (i.e., mother-to-son) transmission events, and $l_{p}$ represents the number of female-to-female (i.e., mother-to-daughter) transmission events in a given path. Note that a female-to-female transmission anywhere in a path will always result in zero genetic contribution from the focal individual to the descendant along that path, as females never pass on their $\mathrm{Z}$ to their daughters. For a given path from a focal individual to a descendant, $h_{p}+j_{p}+k_{p}+l_{p}=g_{p}$. An equivalent way of estimating expected genetic contributions is to simulate the transfer of alleles down the pedigree using gene dropping, emulating random segregation at meiosis ([13]). This method is preferable because it allows us to determine the variance across loci around a calculated expectation and so we use the simulation method here.

To assess the expected autosomal genetic contributions of a single focal individual, we truncated the pedigree above the focal individual to make them a 'founder', i.e., an individual with no known parents in the pedigree. We then assigned 
the focal individual a genotype of '22' and all other founders a genotype of ' 11 ', simulated Mendelian transmission of alleles down the pedigree, and calculated the frequency of the ' 2 ' allele in year $t$. We repeated this process for one million iterations. To assess expected $\mathrm{Z}$ chromosome genetic contributions, we used a similar method, but adjusted transmission rules and assigned females a genotype of ' 2 ' or ' 1 ' for their single copy of the $Z$ chromosome. For this process, we needed to know the sex of all descendants. For the 1,896 unsexed individuals in our population pedigree (all died as nestlings), we took a conservative approach and assigned individuals with missing sex information as male; since males are ZZ, this approach will minimize Z-autosome differences.

We estimated the genealogical and mean expected genetic contributions over time for a set of 926 breeders that bred in the population between 1990 and 2013. These individuals were all born before 2002 and died by the end of 2014, so our estimates should capture all of their offspring as well as some grand-offspring, great-grand-offspring, etc. To test the relationship between an individual's sex and expected individual genetic contributions to the population in 2013, we fit linear models for expected genetic contributions with genealogical contribution, sex, and their interaction as independent variables. We next fit linear models for the ratio of $\mathrm{Z}$ /autosome expected genetic contributions using the expected genetic contribution on the $\mathrm{Z}$ chromosome as the dependent variable and autosomal expected contribution as the independent variable. We also modeled expected genetic contributions on the $\mathrm{Z}$ with autosomal expected contribution, sex, and their interaction as independent variables.

\section{Expected genetic contributions of male and female immigrants}

To quantify the effects of sex-biased migration, we estimated the expected genetic contributions of male and female immigrants for both autosomal and Z-linked loci. Starting with immigrants entering the population in 1991, we assigned all male immigrants a genotype of ' 22 ' and all other founders a genotype of ' 11 ', performed gene dropping using the appropriate transmission rules as described above, and calculated the frequency of the ' 2 ' allele in each year. We repeated this process one million times to find the total expected genetic contributions of all male immigrants as a group. We repeated this process for female immigrants. Note that the total expected genetic contribution of immigrants here is cumulative and includes the contributions of all descendants of immigrants. We used linear models to test for trends in immigration rate and sex ratios of immigrants over time. We included the number of immigrants in our model of sex ratios. Finally, we used linear models to assess the relationship between immigrant cohort size and the expected genetic contribution of that immigrant cohort to the population 15 years later (the longest time period for which we have a large enough data set).

\section{Neutral allele dynamics and selection}

Previous work evaluated signals of selection on 10,731 autosomal loci and detected 18 SNPs with significant changes in allele frequency across 1999-2013 [13]. Here, we tested for selection on the Z chromosome using 249 non-pseudoautosomal SNPs and 19 pseudoautosomal SNPs. To simulate the neutral behavior of Z-linked alleles, we performed gene dropping simulations for each SNP using known founder genotypes. We trimmed the 6936-individual pedigree to remove any founders with missing genotype data for the focal SNP. We re-coded the offspring of trimmed founders as founders, and repeated this process until all founders had genotype data. Thus, the pedigree used for each SNP could vary slightly based on patterns of missing data at that locus. After trimming, we used gene dropping to simulate the transmission of alleles down the pedigree one million times. We used $Z$ chromosome transmission rules for the 249 non-pseudoautosomal Z-linked SNPs, and autosomal transmission rules for the 19 pseudoautosomal loci. From the simulated genotypes in each iteration, we calculated the expected allele frequency shifts using the genotyped nestlings born in each year in a core set of about 68 consistently monitored territories (2841 nestlings in total over 1990-2013). These gene dropping iterations resulted in a distribution of changes in allele frequency over time, conditional on the pedigree. We compared the distribution of allele frequency change expected under neutrality to observed allele frequency changes between 1999 and 2013 and in adjacent years during that time period. We determined p-values for allele frequency change at each SNP by counting the number of simulations in which the simulated value was further from the median of the expected distribution than the observed value. We performed false discovery rate (FDR) correction of p-values for all comparisons across both the autosomes [13] and the Z (10,999 SNPs total) and applied a threshold of FDR $<0.25$ to assess significance.

\section{Model of variance in allele frequencies}

To determine the sources of genome-wide allele frequency change for autosomes and the $\mathrm{Z}$ chromosome, we extended the model from [13] to incorporate the sex of individuals. Like [13], we assumed only three sources of allele frequency change: differences in survival, reproduction (i.e., new births), and immigration among individuals. We classified individuals in our population census each year as survivors, immigrants, or nestlings/births. For this analysis (in contrast to previous analyses), immigrating individuals were only counted as immigrants the year they first appear in the population and were categorized as survivors in following years. We further subdivided each demographic group into males and females in order to account for the possibility that individuals of different sexes contributed differently to each source of allele 
frequency change. We included individuals in the population for a given year if they were observed during at least two months between March and June, and considered individuals who disappeared from the population for one or more years but later returned to have been survivors in the intervening time.

We calculated allele frequency change between consecutive years by contrasting allele frequencies in each category (male survivors, male births, male immigrants, female survivors, female births, female immigrants) in each year with the allele frequency of the entire population in the previous year. Let $N_{t}$ be the total number of individuals in the population in year $t$. Let $N_{M, s}$ be the number of males who survived from year $t-1$ to year $t, N_{M, i}$ be the number of males who immigrated into the population in year $t$, and $N_{M, b}$ be the number of males born in the population in year $t$. Likewise, let $N_{F, s}$ be the number of females who survived from year $t-1$ to year $t, N_{F, i}$ be the number of females who immigrated into the population in year $t$, and $N_{F, b}$ be the number of females born in the population in year $t$. Note that $N_{t}=N_{M, s}+N_{M, i}+N_{M, b}+N_{F, s}+N_{F, i}+N_{F, b}$.

We constructed two models, one for autosomal loci and one for Z-linked loci. Let the allele frequency of a given demographic group $j$ be $p_{M, j}$ for males and $p_{F, j}$ for females, e.g., $p_{F, s}$ is the allele frequency of female survivors. The change in allele frequencies between two adjacent years for an autosomal locus is:

$$
\begin{aligned}
\Delta p_{A}= & \frac{N_{F, s}}{N_{t}}\left(p_{F, s}-p_{t-1}\right)+\frac{N_{F, i}}{N_{t}}\left(p_{F, i}-p_{t-1}\right)+\frac{N_{F, b}}{N_{t}}\left(p_{F, b}-p_{t-1}\right) \\
& +\frac{N_{M, s}}{N_{t}}\left(p_{M, s}-p_{t-1}\right)+\frac{N_{M, i}}{N_{t}}\left(p_{M, i}-p_{t-1}\right)+\frac{N_{M, b}}{N_{t}}\left(p_{M, b}-p_{t-1}\right)
\end{aligned}
$$

Here, the contribution of each category to the overall change in allele frequency over time is a function of the proportion of alleles in the population from that category in year $t$ (equivalent to the porportion of individuals in that category) and the difference in allele frequencies between that category in year $t\left(e . g .\right.$, female survivors, $\left.p_{F, s}\right)$ and the entire population from the year before $\left(p_{t-1}\right)$.

Similarly, the change in allele frequencies between two years for a Z-linked locus is:

$$
\begin{aligned}
\Delta p_{Z}= & \frac{N_{F, s}}{N_{F, t}+2 N_{M, t}}\left(p_{F, s}-p_{t-1}\right)+\frac{N_{F, i}}{N_{F, t}+2 N_{M, t}}\left(p_{F, i}-p_{t-1}\right)+\frac{N_{F, b}}{N_{F, t}+2 N_{M, t}}\left(p_{F, b}-p_{t-1}\right) \\
& +\frac{2 N_{M, s}}{N_{F, t}+2 N_{M, t}}\left(p_{M, s}-p_{t-1}\right)+\frac{2 N_{M, i}}{N_{F, t}+2 N_{M, t}}\left(p_{M, i}-p_{t-1}\right)+\frac{2 N_{M, b}}{N_{F, t}+2 N_{M, t}}\left(p_{M, b}-p_{t-1}\right)
\end{aligned}
$$

Note that males have two copies of the $Z$ while females only have one copy of the $Z$, which impacts the total number of alleles in the population and the relative weighting of male vs. female categories.

For both models, we further partitioned allele frequency change due to births, $\operatorname{Var}\left(p_{M, b}-p_{t-1}\right)$ and $\operatorname{Var}\left(p_{F, b}-p_{t-1}\right)$, into contributions of variation in family size and Mendelian segregation of alleles from heterozygous parents in order to understand the relative roles of these two factors.

Using equations 3 and 4 and accounting for family size and Mendelian noise, we calculated the variance in allele frequencies across autosomal or Z-linked loci in each category, as well as the covariances between categories, e.g., between survival and birth. Pseudoautosomal SNPs were removed in this analysis. We assumed that immigrants in a given year are unrelated to survivors and therefore set all covariances between immigrants and survivors to zero. In addition, for Z-linked loci, female survivors and female immigrants cannot transmit alleles to female nestlings (births), so we also set covariances between female survivors and female nestlings and between female immigrants and female nestlings to zero. We then calculated the proportion of total allele frequency change between a given pair of consecutive years contributed by each variance and covariance term.

While we have a complete census of all individuals in the population, not every individual is genotyped or sexed (Fig. S1). To deal with missing genotype data, we corrected each term for sampling error, estimated empirically via simulations. Briefly, we assigned genotypes to all individuals in the population in 1990 based on an allele frequency drawn from the empirical allele frequency distribution and then simulated Mendelian transmission (of autosomes or of the $\mathrm{Z}$ chromosome) forward in time for 100,000 replicates. For our Z chromosome simulations, we randomly assigned unsexed individuals as males or females based on the empirical sex ratio, which is approximately 50:50. We estimated allele frequencies from the subset of individuals who were genotyped and subtracted the population allele frequency to obtain the sampling error.

We used a bootstrapping approach to generate confidence intervals for our variance and covariance estimates. We sampled SNPs in 3.4 Mb windows along the genome 1,000 times with replacement. A $3.4 \mathrm{Mb}$ window size was chosen to allow at least 1 window per chromosome, as the smallest chromosome in the current Florida Scrub-Jay genome assembly is $3.4 \mathrm{Mb}$. Windows smaller than $3.4 \mathrm{Mb}$, on unplaced scaffolds, or with less than $5 \mathrm{SNPs}$ were discarded. 
Across autosomes, this windowed approach resulted in 299 windows containing between 5 and 257 SNPs, with 50 SNPs/window on average. On the Z, SNPs were split between 21 windows, ranging from 5-83 SNPs. To ensure that our chosen window size $(3.4 \mathrm{Mb})$ was likely to break apart linkage, we estimated levels of linkage disequilibrium (LD) in our study population using the 'ld-window-r2' function in plink [58] to estimate pairwise LD between SNPs in each of our linkage groups. We fit an exponential decay curve for the relationship between pairwise LD and distance between SNPs and found that, on average, $\mathrm{LD}$ decayed around $1 \mathrm{Mb}$ (Fig. S2). We repeated the above sampling and error estimation steps for each window.

We ran an additional set of models that assumed a 50:50 sex ratio for each demographic group (survivors, immigrants, and births). This assumption is fairly reasonable for survivors and births, but not for immigrants (Fig. S1). However, these models allowed us to account for a specific situation in which survivors, who make up a large proportion of the previous year's population, have a similar mean allele frequency to the total allele frequency from last year $\left(p_{t-1}\right)$. When the group of survivors are subdivided into males and females, the mean allele frequency of one group will, by chance, be above $p_{t-1}$, and the mean of the other group below $p_{t-1}$, creating a negative covariance between male and female survivors. By assuming a 50:50 sex ratio in our additional set of models, we were able to compare the allele frequency of each category to the allele frequency of individuals of that sex the previous year (i.e., $p_{M, t-1}$ or $\left.p_{F, t-1}\right)$ and avoid the artifact described above.

Let $N_{M, t}$ be the total number of males in the population in year $t$ and $N_{F, t}$ be the total number of females in the population in year $t$. The change in allele frequencies between two years for an autosomal locus, assuming a 50:50 sex ratio, is:

$$
\begin{gathered}
\Delta p=\left[\frac{1}{2}\right]\left[\frac{N_{M, s}}{N_{M, t}}\left(p_{M, s}-p_{M, t-1}\right)+\frac{N_{M, i}}{N_{M, t}}\left(p_{M, i}-p_{M, t-1}\right)+\frac{N_{M, b}}{N_{M, t}}\left(p_{M, b}-p_{M, t-1}\right)\right] \\
+\left[\frac{1}{2}\right]\left[\frac{N_{F, s}}{N_{F, t}}\left(p_{F, s}-p_{F, t-1}\right)+\frac{N_{F, i}}{N_{F, t}}\left(p_{F, i}-p_{F, t-1}\right)+\frac{N_{F, b}}{N_{F, t}}\left(p_{F, b}-p_{F, t-1}\right)\right]
\end{gathered}
$$

For the full derivation of the autosomal model and for the model for Z-linked loci, see Appendix B of the supplementary materials.

\section{Implementation}

We implemented gene dropping in python and $\mathrm{C}++$ and used this software to conduct the simulations for expected genetic contributions and tests of selection. We performed analyses and visualization in R v. 3.6.3 [59] using packages base, stats, plyr [60], dplyr [61], ggplot2 [62], cowplot [63], and kinship2 [64].

We implemented the allele frequency variance models in R v. 4.1.1 [59] using packages base, stats, foreach [65], doParallel [66], plyr, and dplyr. We used the packages ggplot2 and cowplot for visualization.

\section{Data Availability}

Scripts are available on github and data are available on Figshare [links to come].

\section{Results}

\section{Expected genetic contributions of males and females}

We first focused on the contributions of individual breeding adults to future generations. Each individual breeder has both a genealogical and expected genetic contribution to the future. Here, we define the genealogical contribution of an individual as the proportion of a given birth cohort that is descended from that individual. In contrast, the expected genetic contribution of an individual is the expected proportion of alleles in a given birth cohort inherited identicalby-descent from our focal individual. An individual's expected genetic contribution differs from their genealogical contribution because not every chromosome is passed down to the next generation. Expected genetic contributions are also impacted by transmission rules; sex-biased transmission of the $\mathrm{Z}$ chromosome results in different genetic contributions of individuals at autosomal versus $\mathrm{Z}$ loci.

To illustrate the effect of sex-biased transmission on individual genetic contributions across the genome, we contrasted the expected genetic contributions for autosomal and Z-linked loci over time for a male and female who are each others' exclusive mates. This pair first bred in 2001, produced 15 offspring together, and had a total of 223 descendants by 2013 (Fig 1A). Since these breeders did not pair with any other individuals over the course of their lives, they have the same descendants and thus equal genealogical contributions. At a given autosomal locus, the male and female had equal expected genetic contributions (Fig 1B). The only differences in the expected autosomal genetic contributions of the male and female arose from the stochastic nature of the simulations we used to estimate expected genetic contributions; 
A

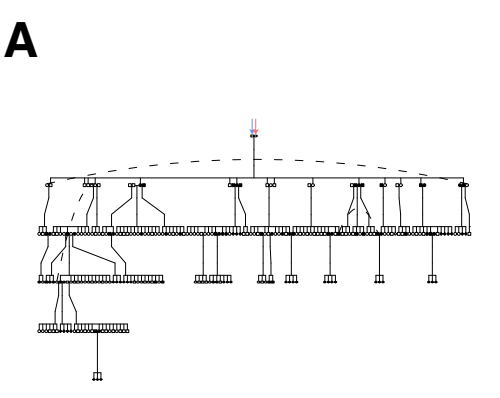

after 1,000,000 iterations, their values were essentially identical. In contrast, the expected genetic contributions on the Z chromosome tended to be considerably higher for the male than for the female between 2005 and 2013 (Fig. 1C).
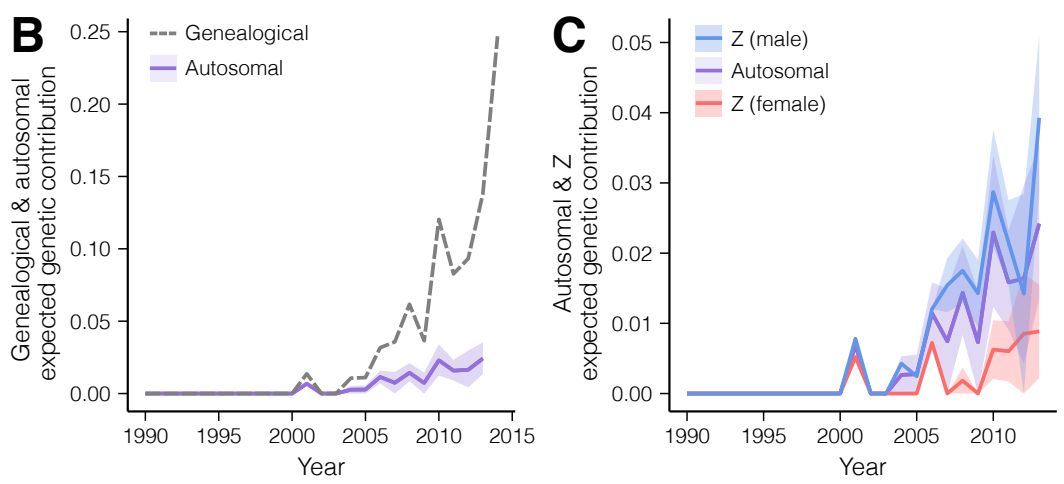

Figure 1 (A) Pedigree of descendants and (B, C) genealogical and genetic contributions over time for a male-female pair that first bred in 2001 with total lifetime reproductive success of 15 . In A, dashed lines connect individuals who appear more than once in the pedigree. In B, dashed lines indicate the proportion of nestlings in each cohort who are genealogical descendants of the pair. Solid lines indicate the mean expected genetic contribution at a neutral autosomal or Z chromosome locus for each year, and pale shading indicates the $95 \%$ confidence interval. Expected autosomal genetic contributions are shown in purple, and expected $\mathrm{Z}$ genetic contributions of the male partner are shown in blue and of the female in red.

Moving to the population level, we compared the genealogical and mean expected genetic contributions to our study population in 2013 of a set of 926 individuals who bred in our study population between 1990 and 2013. In this set of breeders, 317 males and 340 females have 0 descendants by 2013. For breeders with a least one descendant in 2013, genealogical contributions to the study population in 2013 ranged from 0.00337 to 0.313 (mean $=0.0534$ ) for males and from 0.00337 to 0.313 (mean $=0.0544$ ) for females. The similar range of genealogical contributions between males and females was unsurprising given this species is monogamous and the mortality rate is the same between the sexes [53,67]. Expected genetic contributions for an autosomal locus ranged from 0.000420 to 0.0242 (mean $=0.00492$ ) for males and from 0.000420 to 0.0242 (mean $=0.00488$ ) for females. Expected genetic contributions for a $\mathrm{Z}$ chromosome locus ranged from 0 to 0.0393 (mean $=0.00658$ ) for males and from 0 to 0.0207 (mean $=0.00368)$ for females.

We found that for an autosomal locus, the relationship between genealogical and expected genetic contributions did not significantly differ for males versus females (Fig 2A; genealogical contribution: $p<2 \mathrm{e}-16$; sex: $p=0.82$; genealogical ${ }^{*}$ sex : $p=0.16)$. For a $\mathrm{Z}$ chromosome locus, on the other hand, the relationship between genealogical and expected genetic contributions for males versus females was strikingly different (Fig 2B; genealogical contribution: $p<2 \mathrm{e}-16$; sex: $p=0.26$; genealogical ${ }^{*}$ sex $: p<2 \mathrm{e}-16$ ). The genealogical estimate for females (genealogical ${ }^{*}$ sex: $\beta=0.040$, Standard Error (SE) $=0.0044$ ) was $48 \%$ lower than the genealogical estimate for males (genealogical ${ }^{*}$ sex: $\beta=0.083, \mathrm{SE}=0.0032$ ). For both autosomal and $\mathrm{Z}$ chromosome loci, expected genetic contributions were much lower than genealogical contributions, consistent with theoretical predictions (the gray dotted lines in Figs 2A and 2B show a 1:1 relationship).

We directly compared the expected genetic contributions of an autosomal locus and a Z locus. Mathematically, the ratio of male $\mathrm{Z}$ /autosome expected genetic contributions is $\frac{4}{3}$ while female $\mathrm{Z}$ /autosome expected genetic contributions is $\frac{2}{3}$ (See Appendix A). When sex is not considered, average expected genetic contributions to $Z$ and autosomal loci should be equal because we defined expected genetic contribution as a proportion of the total number of $Z$ chromosomes or autosomes respectively. As anticipated, the ratio of $\mathrm{Z}$ /autosome expected genetic contributions of individuals without regard to sex was close to 1 (slope $=0.97, \mathrm{SE}=0.018$ ). When sex was added to the model (which explained $17 \%$ more variance than the model without sex), the $\mathrm{Z}$ /autosome ratio of expected genetic contributions was significantly impacted by sex $(p<2 \mathrm{e}-16)$; the $Z$ /autosome estimate for females $(0.6747, \mathrm{SE}=0.034)$ was lower than the $Z$ /autosome estimate for males $(1.25, \mathrm{SE}=0.024)$ by $54 \%$. The $\mathrm{Z}$ / autosome ratio of expected genetic contributions for females matched our expectations $\left(\frac{2}{3}\right)$, while the $\mathrm{Z}$ /autosome ratio for males was slightly less than the expected $\frac{4}{3}$ (Fig. 2C).

We further explored the relationship between genealogical and expected genetic contributions for autosomes and the $\mathrm{Z}$ chromosome by comparing the contributions of male and female partners. While Florida Scrub-Jays are monogamous, many individuals pair again if their partner dies. For the small subset of pairs that only bred with each other throughout their entire life (i.e., lifelong monogamous pairs), the male and female partners always had equal expected autosomal genetic contributions in 2013 (Fig S3A), but some males had higher expected Z genetic contributions than their mate, especially when the sex ratio of their offspring was skewed towards female offspring (Fig. S3B). Recall that mothers only 


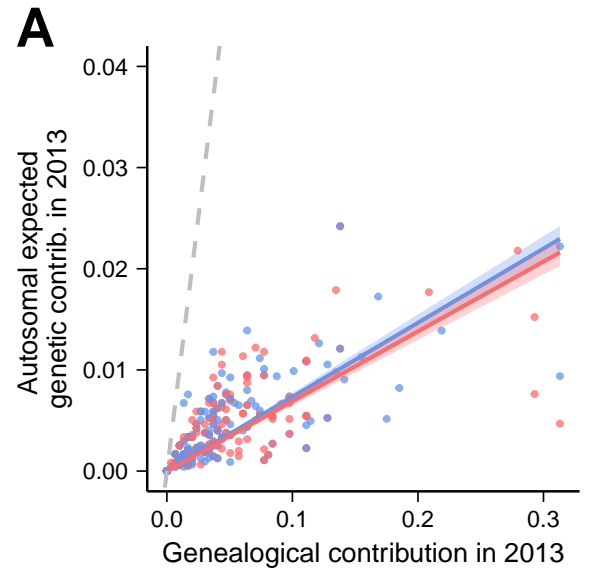

transmit their $Z$ chromosomes to their sons and therefore do not contribute $Z$ alleles to their daughters or any descendants of their daughters. We examined the pedigrees of all descendants for the six lifelong monogamous pairs with unequal expected $Z$ genetic contributions between partners. The three female breeders with zero expected $Z$ genetic contributions in 2013 had only daughters that contributed to the 2013 birth cohort, while the three female breeders with greater than zero expected Z genetic contributions had descendants in the 2013 birth cohort via both sons and daughters. The fact that Florida Scrub-Jays can have multiple mates throughout their lifetime may increase the difference in expected genetic contributions between male-female pairs. To confirm this hypothesis, we compared the expected genetic contributions of all male and female partners and tracked the number of mates each individual had throughout their lifetime (Fig. S3C and D). For breeding pairs in which the female breeder had more mates throughout her lifetime compared to the male breeder, the female tended to have higher expected autosomal genetic contributions than her mate, and the opposite was true if the male breeder had a higher number of mates. We found a similar pattern for the $\mathrm{Z}$ chromosome. However, many females had lower expected $\mathrm{Z}$ genetic contributions than their male partners despite having a higher number of mates because of the important role the sex ratio of descendants plays in determining expected $Z$ genetic contributions.
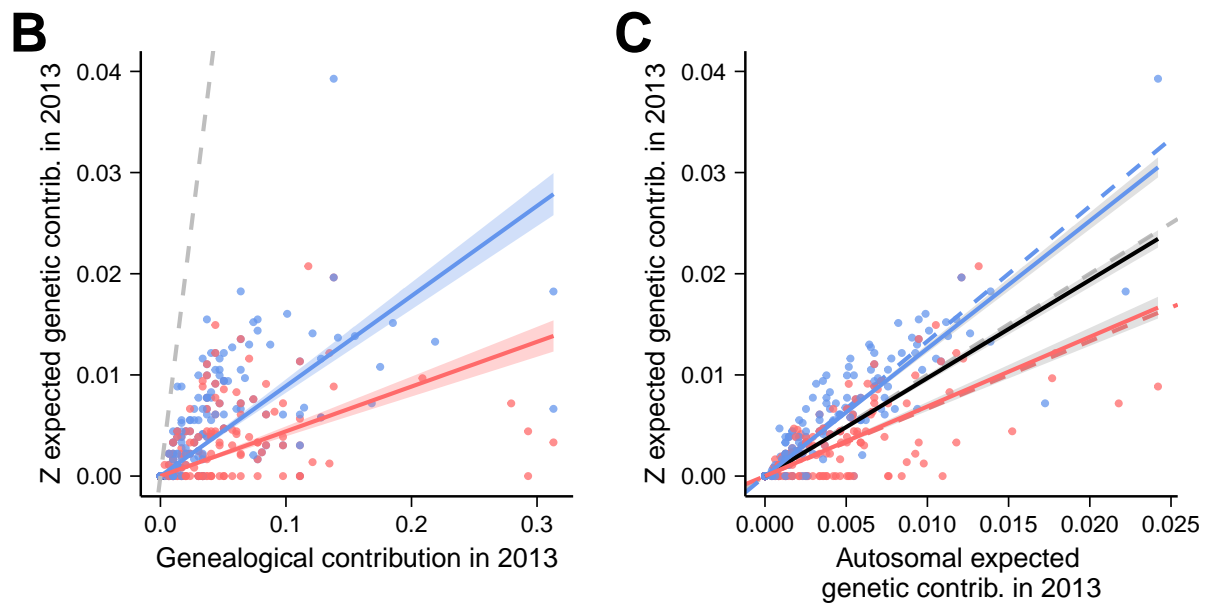

Figure 2 Genealogical and expected genetic contributions to the population in 2013 at (A) a neutral autosomal and (B) a neutral $\mathrm{Z}$ chromosome locus for all male breeders $(n=351$, blue) and female breeders $(n=378$, red) born before 2002 who bred in the population between 1990 and 2013. The dotted lines indicate a one-to-one relationship. (C) Expected genetic contributions to the population in 2013 at a neutral autosomal locus versus expected genetic contributions to the population in 2013 at a neutral Z chromosome locus for the breeders shown in parts A and B. The black line shows the Z/autosome relationship without regard for sex, the blue line shows the Z/autosome relationship for males and the red line shows the $\mathrm{Z}$ /autosome relationship for females. Solid lines show estimates from linear models for each group, and dashed lines show theoretical expectations (See Appendix A).

\section{Expected genetic contributions of immigrants}

Florida Scrub-Jays have female-biased dispersal, and previous work showed high levels of immigration into our study population over time [13, 54]. Between 1990 and 2012, the number of immigrant breeders appearing in Archbold each year was generally small (6-40/year; Fig. 3A). The immigrant breeder sex ratio was female-biased in 16 of 22 years (mean proportion of male immigrants across years $=0.39)$. Immigration decreased significantly over time $(-0.46 \pm 0.065$ immigrants /year, $R^{2}=0.32$, $\left.p_{\text {immigrant }}=3.23 \mathrm{e}-10\right)$, and sex was not a significant factor in this change $\left(p_{\text {sex }}=0.231\right)$.

To investigate the role of sex-biased migration, we calculated the expected genetic contributions of male and female immigrants who entered the population between 1990 and 2013. Female immigrants had significantly higher expected genetic contributions at autosomal loci than male immigrants by 2013 (Fig. 3B; significance based on non-overlapping $95 \%$ confidence intervals). However, despite female-biased immigration rates, the expected genetic contribution of male immigrants at Z-linked loci was significantly higher than that of female immigrants until 2011 (Fig. 3C). Consistent with previous work [13], 75\% of the autosomal alleles in the 2013 birth cohort were contributed by immigrants arriving since $1990,62 \%$ of which was driven by female immigrants. On the $Z$ chromosome, the total expected genetic contribution of male and female immigrants was also 75\% in 2013 (Fig. 3C), with each sex contributing half (50\%) of the incoming alleles. When we normalized expected $Z$ genetic contributions by expected autosomal genetic contributions (thus accounting for sex-biased immigration), the $\mathrm{Z}$ /autosome ratio approached $\frac{4}{3}$ for male immigrants and $\frac{2}{3}$ for female immigrants, as expected (Fig. S4). 
bioRxiv preprint doi: https://doi.org/10.1101/2021.10.28.466320; this version posted October 29, 2021. The copyright holder for this preprint (which was not certified by peer review) is the author/funder, who has granted bioRxiv a license to display the preprint in perpetuity. It is made available under aCC-BY-NC 4.0 International license.

While all immigrants could have sustained genetic contributions to a population over time, gene flow could alternatively be driven by a few immigrant individuals with large contributions, in which case immigrant cohort size would not be a good predictor of gene flow. To this end, we assessed the relationship between immigrant cohort size and total expected genetic contribution of that immigrant cohort to individuals born 15 years later. We found that immigrant cohort size was a good predictor of autosomal (Fig 3D) and Z-linked (Fig 3E) expected genetic contributions. At autosomal loci, we found that the expected genetic contribution of a given immigrant cohort significantly increased with increasing cohort size, but there was no significant effect of sex on the rate of increase $\left(\beta=0.0025 \pm 0.0008, R^{2}=0.35, p_{\text {slope }}=0.012\right.$, $\left.p_{\text {sex }}=0.34\right)$. Immigrant sex had an impact for Z-linked loci: immigrant cohort size was not significantly associated with expected Z genetic contribution after 15 years for female immigrants $(\beta=0.0016 \pm 0.00095, p=0.1199)$ but was significant for male immigrants $\left(\beta=0.019 \pm 0.0084, p=0.041\right.$; full model $\left.R^{2}=0.25\right)$. Overall, we saw a similar relationship between immigrant cohort size and expected genetic contributions of male and female immigrant cohorts on the autosomes but our results suggest male immigrant cohort size has larger impact than female immigrant cohort size on expected genetic contributions on the Z.

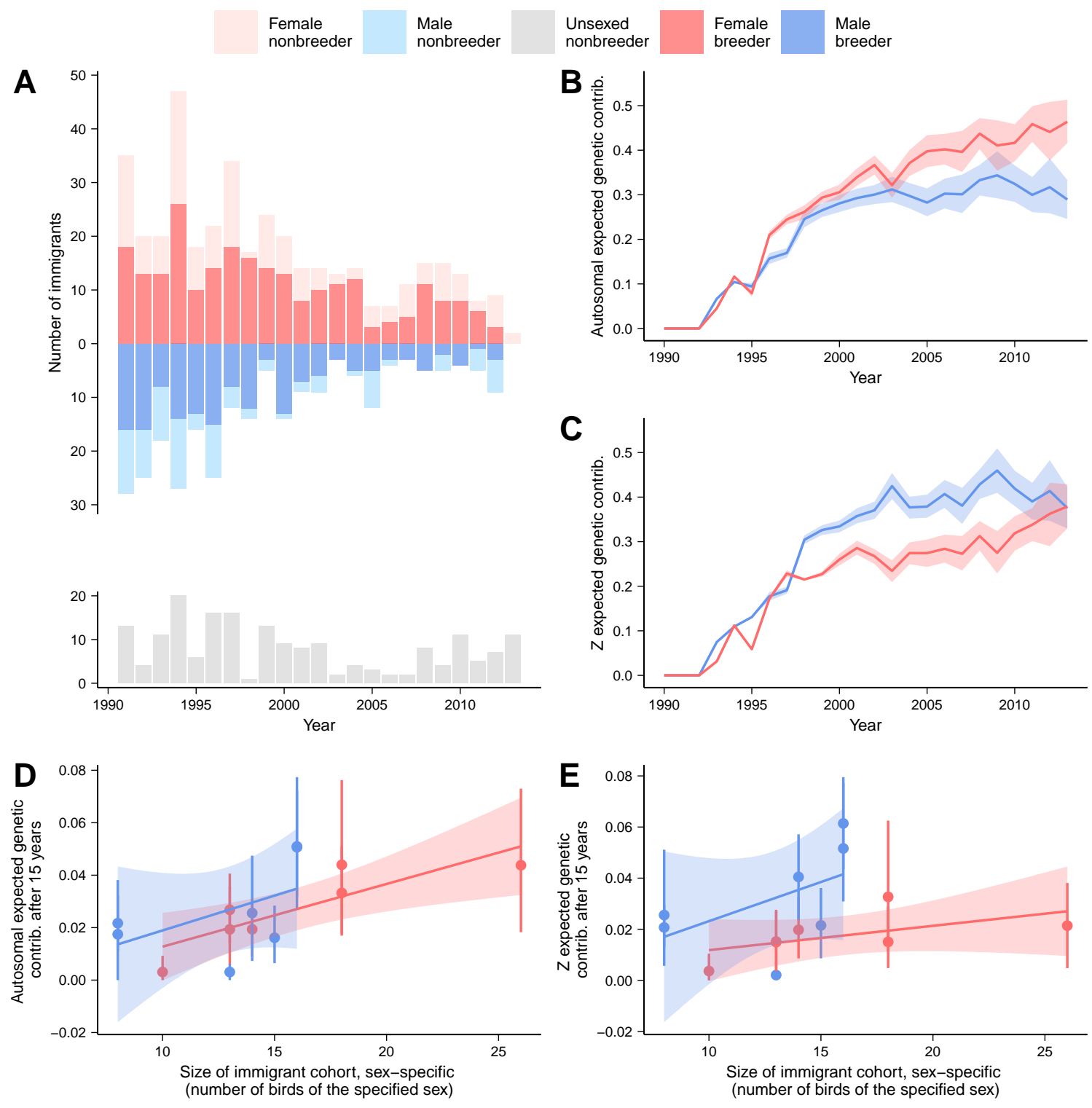

Figure 3 (A) The number of female immigrants (shown in light/dark red for nonbreeders/breeders), male immigrants (shown in light/dark blue for nonbreeders/breeders), and immigrants of unknown sex (all nonbreeders, shown in gray) arriving in the Archbold population each year between 1990 and 2013. (B, C) The expected genetic contribution of male and female immigrants appearing in the population after 1990 at (B) a neutral autosomal locus and (C) a neutral Z chromosome locus. (D, E) The expected genetic contribution of immigrant cohorts between 1991 and 1997 to the nestling cohort 15 years later at (D) a neutral autosomal locus and (E) a neutral $\mathrm{Z}$ chromosome locus. 


\section{Signals of Selection}

We next evaluated signals of selection on 249 Z-linked loci and 19 SNPs in the PAR (which follows autosomal transmission patterns) by comparing the change in allele frequencies expected under the pedigree to the observed change [13]. We identified loci at which the observed change in allele frequency between 1999 and 2013 was larger than expected from our simulations as likely to be under directional selection. Across 1999-2013, we found a single Z-linked SNP that showed significantly larger shifts in allele frequency than would be expected from gene flow and drift alone at a FDR of 0.25 (Fig. S5). We also compared pairs of consecutive years (e.g., 1999 and 2000, 2000 and 2001, etc.) and found a total of 4 Z-linked SNPs - 1 from the 1999-2000 comparison and 3 from the 2000-2001 comparison-that showed significantly more allele frequency change than would be expected from gene flow and drift alone from one year to the next at a FDR of 0.25 (Fig. S6). All significant loci were Z-linked; we found no significant sites among the 19 PAR SNPs. We reassessed the 10,731 autosomal loci examined by [13] after correcting for multiple comparisons across both autosomal and $\mathrm{Z}$ loci and found results were unchanged except, in the consecutive year comparisons, 4 autosomal SNPs-1 from the 2001-2002 comparison and 3 from the 2003-2004 comparison-which were previously significantly different between years are no longer significant (FDR $>0.25$ ).

\section{Drivers of allele frequency change}

To quantify the relative roles of different evolutionary processes, we partitioned genome-wide allele frequency change between consecutive years among demographic groups (survivors, immigrants, nestlings/births; Fig. 4A), assuming survival/reproduction and gene flow are the only sources of allele frequency change (which is true for neutral alleles). Building on the model of [13], we additionally accounted for and assessed sex-specific demography and sex-specific inheritance by allowing differences between males and females in each demographic group (Fig. 4A) and creating separate models that use the transmission rules for the $\mathrm{Z}$ chromosome.

We first looked at the relative contributions of males, females, and male-female covariance to overall allele frequency change (Fig. 4B). For autosomes, the proportion of variance contributed by males and by females over years was generally similar, ranging from 0.34 to 0.60 for males and from 0.40 to 0.55 in females. Males had a similar contribution to variance in allele frequencies on the $\mathrm{Z}(0.42$ to 0.60$)$ while females contributed proportionally less to total allele frequency change on the Z (0.19 to 0.45$)$ than on the autosomes. We also looked at the relative contributions of survivors, immigrants, births, and covariances between groups to overall allele frequency change (Fig. 4C). At autosomal loci, our findings were similar to the analysis of [13], with survivors, births, and survivor-birth covariance consistently contributing between 84 and $98 \%$ of the variance. Between 88 and $99 \%$ of the variance in allele frequencies on the $\mathrm{Z}$ chromosome was also due to variation in survival, reproduction or survivor-birth covariance.

Next, we investigated the contributions of each sexed demographic group (Fig. 5). At autosomal loci, there was significant overlap in our confidence intervals between male and female survivors, suggesting both contribute similarly to allele frequency change from year to year. At Z-linked loci, male survivors tended to contribute more to variance in allele frequencies than females, but confidence intervals overlapped. We calculated the ratio between contributions to $Z$ and autosomal allele frequency change and found that the $\mathrm{Z}$ /autosome ratio for female survivors approximated the expected $\frac{2}{3}$, while the $\mathrm{Z}$ /autosome ratio for male survivors was more similar to $\frac{1}{1}$ (Fig. S7).

The covariance between male and female survivors declined across years on the $Z$, although it was positive in the first few years of the study period and confidence intervals overlapped zero in all years. Covariance between male and female survivors was consistently negative at autosomal loci, though confidence intervals again overlapped zero in all years (Fig. 5). The observed negative covariance could be a mathematical artifact arising because survivors were a large proportion of the population from the previous year (see Methods). Briefly, a negative covariance could arise simply by splitting the allele frequency of the whole population in year $t-1$ into separate sexed groups in year $t$. We confirmed this hypothesis by running a second set of models that compared allele frequencies between males/females in each demographic group with the sex-specific population allele frequency from the previous year. These models did not have the same biases based on properties of the mean, and indeed we found near-zero covariances between male and female survivors (Fig. S8).

Confidence intervals for the variance in allele frequency change due to male and female births overlapped for autosomal loci. On the $\mathrm{Z}$, male births tended to play a larger role than female births, but confidence intervals overlapped here as well. For female births, the ratio between contributions to $Z$ and autosomal allele frequency change was around $\frac{2}{3}$, while for male births the ratio was around $\frac{4}{3}$ (Fig. S7). We further separated the variance due to births into contributions of Mendelian segregation in heterozygous individuals and of variation in family size (Fig. S9). The ratio between contributions to $Z$ and autosomal allele frequency change was approximately $\frac{2}{3}$ for both female and male Mendelian noise (Fig. S10). For female family size, the Z/autosome ratio was $\frac{2}{3}$, while for male family size the ratio was slightly above $\frac{4}{3}$. The covariance between male and female births was not significantly different from zero for both autosomes and the $\mathrm{Z}$. 


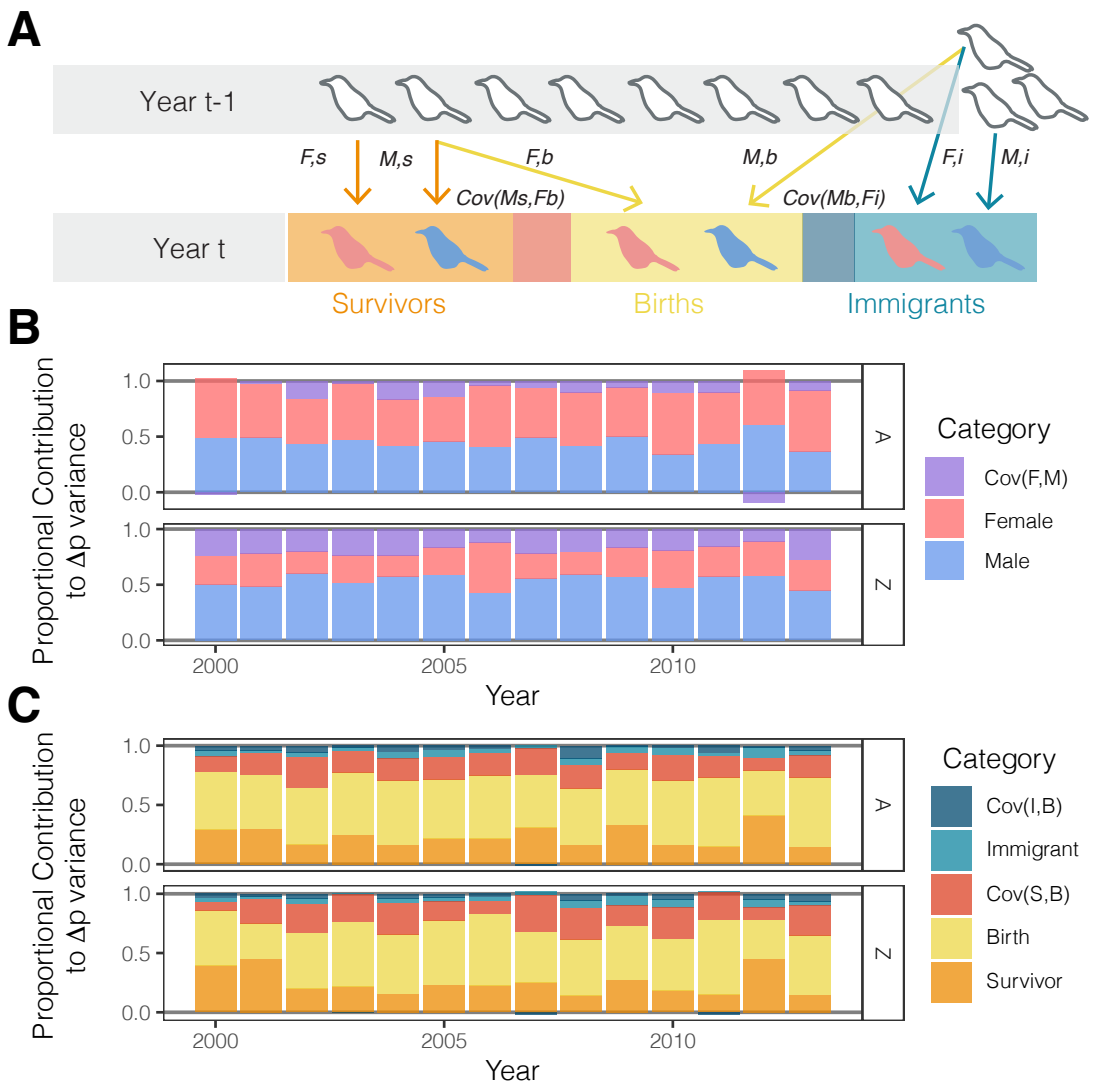

Figure 4 (A) Schematic of the allele frequency variance partitioning model. Arrows show contributions to allele frequency change through different demographic processes: survival, reproduction (birth), and immigration. $F, s=$ female survivors; $M, s=$ male survivors; $F, b=$ female births; $M, b=$ male births; $F, i=$ female immigrants; and $M, i=$ male immigrants. (B, C) Results of the model. (B) Allele frequency variance partitioning for males (blue), females (pink), and covariance between males and females (purple) for autosomal loci (top) and Z-linked loci (bottom). (C) Allele frequency variance partitioning for survivors (orange), births (yellow) and immigrants (teal), as well as covariance between survivors and births $(\operatorname{Cov}(\mathrm{S}, \mathrm{B})$, red) and covariance between immigrants and births ( $\operatorname{Cov}(\mathrm{I}, \mathrm{B})$, blue) for autosomal loci (top) and Z-linked loci (bottom).

We found that, per year, immigration played a small role in allele frequency change (-0.0026 to 0.056$)$, about an order of magnitude less than births (-0.088 to 0.50$)$ or survival (-0.23 to 0.40$)$. At autosomal loci, female immigrants tended to contribute more than males, although the confidence intervals overlapped. On the $Z$, male immigrants contributed more than females. The $\mathrm{Z}$ /autosome ratio for male immigrants was well above $\frac{4}{3}$, while the $\mathrm{Z}$ /autosome ratio for female immigrants was much lower than $\frac{2}{3}$ (Fig. S7).

\section{Discussion}

The use of temporal samples to understand allele frequency change over time is a growing area of research in evolutionary biology. Here, we investigated the effects of sex-biased demography, sex-specific inheritance, and their interplay on expected genetic contributions and allele frequency change over short timescales. Using expected genetic contributions obtained by simulating the transmission of alleles down the pedigree, we found similar average contributions of the sexes on autosomes, but highly male-biased contributions to the $\mathrm{Z}$ chromosome. We also partitioned the proportion of allele frequency change among consecutive years due to sex-specific survival/reproduction and gene flow. Consistent with previous work [13], we found that overall, differential birth and survival drives the majority of allele frequency change. While contributions to allele frequency change at autosomal loci are equally distributed between the sexes, males contribute more to allele frequency change at Z-linked loci than females. Together, our results offer unique insights into the impacts of sex-specific processes on evolution over ecologically relevant timescales.

Here, we estimated an individual's expected genetic contribution as the expected proportion of alleles in each birth cohort inherited identical-by-descent from the focal individual. After 10 generations, the expected genetic contributions 


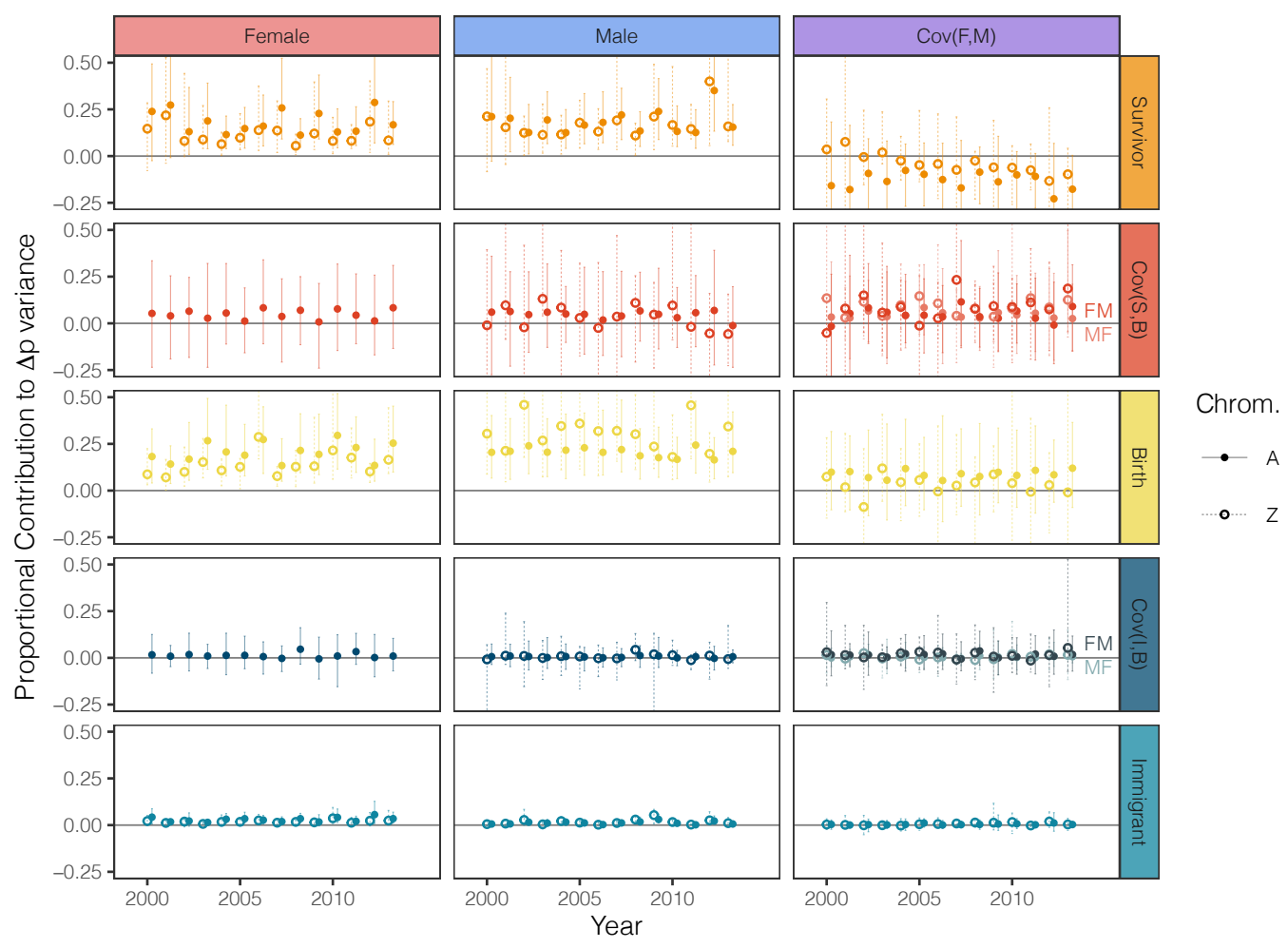

Figure 5 Contributions to the variance in allele frequency change across years of three demographic processes among the sexes. Solid circles and solid lines show the estimates for autosomal loci; open circles and dotted lines show the estimates for Z-linked loci. Vertical bars show 95\% confidence intervals from bootstrapping. Cov(S,B) indicates covariance between survivors and births, and $\operatorname{Cov}(\mathrm{I}, \mathrm{B})$ indicates covariance between immigrants and births. In the plot showing the covariance between survivors and births of different sexes, the lines labeled "MF" indicate $\operatorname{cov}($ male survivors, female births) and the lines labeled "FM" indicate cov(female survivors, male births); likewise for the covariance between immigrants and births of different sexes.

of an individual will stabilize across years at the reproductive value for that individual $[15,68,69]$. An individual's reproductive value, a concept first introduced by Fisher [10] as a way of understanding allele frequency change in agestructured populations, is obtained from an individual's relative contribution to the future gene pool and is an accurate measure of fitness $[15,70]$. Reproductive values are traditionally estimated in population ecology as a weighted average of present and future reproduction by an individual at a given age [71]. While the reproductive value is a highly accurate measure of an individual's fitness, the expected genetic contributions of an individual captures both differences in fitness between individuals but also chance changes in demography on very short timescales [72]. Given that Florida Scrub-Jays have an estimated generation time of 5 years (Nguyen et al., in prep.), we expect an individual Florida Scrub-Jay's expected genetic contribution to converge to its reproductive value within 50 years [15]. We found that the ratio of expected genetic contributions for autosomes and $\mathrm{Z}$ hover around $\frac{2}{3}$ for females and $\frac{4}{3}$ for males, suggesting that the expected genetic contributions of groups (e.g., all individuals of a given sex) can stabilize on much shorter timescales than the expected genetic contributions of individuals (Appendix A). Our work supports our growing theoretical understanding of differences in expected genetic contributions [73] and reproductive values on sex chromosomes [29, 74]. Further understanding and quantifying the relationship between reproductive value on autosomes versus $\mathrm{Z}$ chromosomes is an important question for future work.

To quantify the contributions of different evolutionary processes and sexes to the variance in allele frequency change from year to year, we partitioned the variance in allele frequency change among sexes and demographic groups for autosomes and the $\mathrm{Z}$ chromosome. For the majority of the terms in our model, males contributed $\frac{4}{3}$ as much on the $\mathrm{Z}$ chromosome compared to autosomes, and females contributed $\frac{2}{3}$ as much on the $\mathrm{Z}$ compared to autosomes. These ratios are strikingly similar to those expected from the differences in effective population size between autosomal and sex-linked regions of the genome [25]. In fact, our results suggest that sex-biased survival or reproductive success have little impact on allele frequency change in the Florida Scrub-Jay; the only potentially sex-biased process is immigration. Indeed, the Florida Scrub-Jay is a simple case study from a population genetic standpoint. Florida Scrub-Jays are monogamous 
with a 50:50 breeder sex ratio and equal variance in reproductive success for males and females [49]. Nonetheless, some terms in our allele frequency partitioning model departed from the $\frac{2}{3}$ and $\frac{4}{3}$ trend. First, when we separated allele frequency change due to births into the contributions of family size variation and Mendelian (random) assortment of chromosomes, the role of random assortment on the $\mathrm{Z}$ was $\frac{2}{3}$ that of the autosomal term for both males and females (Fig. S10). This observation is to be expected as, for $Z$ chromosomes, only one assortment event occurs per parent-offspring triad (during transmission from father to offspring), and thus all offspring regardless of sex have reduced change in allele frequencies on the $\mathrm{Z}$ chromosome compared to autosomes that is due to random of assortment of chromosomes. Second, the proportion of allele frequency change contributed by the male survival term has a 1:1 Z/autosome ratio, which is lower than other ratios. There are several sources of variation that might impact this correlation, most notably the allele frequencies themselves, so we expect this correlation to be complex and are not presently able to pinpoint why this ratio is $1: 1$. Third, we observed no correlation between allele frequency change contributed by female immigrants on the $Z$ and allele frequency change contributed by female immigrants on autosomes, in contrast to a strong correlation between $\mathrm{Z}$ and autosomal allele frequency variance in most categories. Future work could focus on the variation in $\mathrm{Z}$ /autosome ratios between demographic groups discovered here.

The Florida Scrub-Jay offers a useful test of the impact of sex-biased migration rates on short-term allele frequency dynamics: in our study population of jays, even though new immigrants (i.e., immigrants that just arrived that year) only make up a small proportion of the census population size in any given year, nearly a quarter of breeders in each year were once immigrants. Previous work on these jays [51] found higher identity-by-descent for the $Z$ chromosome compared to the autosomes at short distances due to both a lower effective population size of the $\mathrm{Z}$ and female-biased dispersal. Here we showed that, due to sex-biased immigration, female immigrants have higher autosomal contributions than male immigrants. However, due to sex-biased ploidy and transmission of the $Z$, male immigrants had higher $Z$ contributions than female immigrants, consistent with expectations.

Our results also offer insights into the impact of sex-specific differences in variance in reproductive success. By following changes in allele frequency between years separately for males and females, we evaluated the impact of differences in life history between the sexes and their covariance on allele frequencies on the $Z$ and autosomes. The general increase in the covariance between males and females on the $Z$ compared to the autosomes is likely due to differences in transmission rules: because mothers do not transmit a $\mathrm{Z}$ chromosome to their daughters, daughters are more likely to share alleles with their brothers on the $Z$ than on autosomes. However, by breaking down the contributions of covariances between males and females for survival and birth (reproduction) to allele frequency change, we may be able to evaluate whether sex-biases in reproductive success result in allele frequency change [75]. In the Florida Scrub-Jay, we find the negative covariance in survival between the sexes is best explained by random changes in the adult sex ratio between years rather than by a systematic difference between the sexes. Future application of our method to systems with more complex mating systems, life histories, and demographies is likely to be highly informative of the propensity of sexual selection and sexual conflict to impact allele frequency changes on short timescales.

While this study employed $\sim 250$ Z-linked SNPs, we expect we effectively captured the history of the the Z chromosome in the Florida Scrub-Jay because the average distance between our SNPs is smaller than the average breakdown in linkage disequilibrium. However, our analysis of variance on the $Z$ yielded large bootstrap intervals, so denser genotype data would give us more power to detect significant differences. Additionally, while we included the 19 PAR SNPs in our tests of selection, we do not include the PAR in our analysis of expected genetic contributions on the Z, nor that of allele frequency change on the $Z$; expected genetic contribution simulations for the $Z$ were performed using the transmission rules for Z-linked loci not in the PAR. Given the unique evolutionary trajectory of pseudoautosomal alleles [76], future work could expand on our analysis of the PAR.

Overall, we showed that both sex-biased demography and sex-biased transmission had a strong impact on Z chromosome dynamics in a population of Florida Scrub-Jays. We found that proportional contributions of males and females to $\mathrm{Z}$ chromosomes compared to autosomes follow a straightforward $\frac{4}{3}-\frac{2}{3}$ pattern in nearly every case, even on a relatively short evolutionary timescale. Our Beadchip dataset only captures common, variant sites; ongoing whole genome resequencing efforts will allow us to look at invariant sites and therefore tackle estimates of genetic diversity and test for a $\frac{3}{4} \mathrm{Z}$ /autosome diversity pattern. In addition, future work that traces the inheritance of haplotypes down the pedigree as opposed to single SNPs would allow the characterization of actual, realized genetic contributions of individuals across the genome and provide a more detailed picture of how sex-biased demography and sex-biased transmission influence short-term evolutionary dynamics.

\section{Acknowledgments}

We would like to thank Graham Coop for his help with developing the allele frequency partitioning model and the Chen and Coop labs for feedback on the manuscript. Thank you to the many students and staff at Archbold Biological Station who collected field data. We acknowledge funding from the National Science Foundation (NSF grants DEB0855879 and 
DEB1257628) and National Institutes of Health (NIH grant R35GM133412). R.M.H.D. was supported by NSF Graduate Research Fellowship 1939268, and F.E.G.B. was supported by NIH grant R35GM133412.

\section{References}

1. Hairston Jr, N. G., Ellner, S. P., Geber, M. A., Yoshida, T. \& Fox, J. A. Rapid evolution and the convergence of ecological and evolutionary time. Ecol. Lett. 8, 1114-1127 (2005).

2. Carroll, S. P., Hendry, A. P., Reznick, D. N. \& Fox, C. W. Evolution on ecological time-scales. Funct. Ecol. 21, 387-393 (2007).

3. Schoener, T. W. The newest synthesis: understanding the interplay of evolutionary and ecological dynamics. Science 331, 426-429 (2011).

4. Messer, P. W., Ellner, S. P. \& Hairston Jr, N. G. Can Population Genetics Adapt to Rapid Evolution? Trends Genet. 32, 408-418 (2016).

5. Pennings, P. S. Standing genetic variation and the evolution of drug resistance in HIV. PLoS Comput. Biol. 8, e1002527 (2012).

6. Losos, J. B. et al. Evolutionary biology for the 21st century. PLoS Biol. 11, e1001466 (2013).

7. Eizaguirre, C. \& Baltazar-Soares, M. Evolutionary conservation-evaluating the adaptive potential of species. Evol. Appl. 7, 963-967 (2014).

8. Kreiner, J. M. et al. Multiple modes of convergent adaptation in the spread of glyphosate-resistant Amaranthus tuberculatus. Proc. Natl. Acad. Sci. U. S. A. 116, 21076-21084 (2019).

9. Wright, S. Systems of Mating. I. The biometric relations between parent and offspring. Genetics 6, 111-123 (1921).

10. Fisher, R. A. The genetical theory of natural selection (1930).

11. Bergland, A. O., Behrman, E. L., O’Brien, K. R., Schmidt, P. S. \& Petrov, D. A. Genomic evidence of rapid and stable adaptive oscillations over seasonal time scales in Drosophila. PLoS Genet. 10, e1004775 (2014).

12. Therkildsen, N. O., Wilder, A. P., Conover, D. O., Munch, S. B., Baumann, H. \& Palumbi, S. R. Contrasting genomic shifts underlie parallel phenotypic evolution in response to fishing. Science 365, 487-490 (2019).

13. Chen, N., Juric, I., Cosgrove, E. J., Bowman, R., Fitzpatrick, J. W., Schoech, S. J., Clark, A. G. \& Coop, G. Allele frequency dynamics in a pedigreed natural population. Proc. Natl. Acad. Sci. U. S. A. 116, 2158-2164 (2019).

14. Chang, J. T. Recent common ancestors of all present-day individuals. Adv. Appl. Probab. 31, 1002-1026 (1999).

15. Barton, N. H. \& Etheridge, A. M. The relation between reproductive value and genetic contribution. Genetics 188, 953-973 (2011).

16. Arnold, S. J. Bateman's principles and the measurement of sexual selection in plants and animals. Am. Nat. 144, S126-S149 (1994).

17. Janicke, T., Häderer, I. K., Lajeunesse, M. J. \& Anthes, N. Darwinian sex roles confirmed across the animal kingdom. Sci Adv 2, e1500983 (2016).

18. Li, X.-Y. \& Kokko, H. Sex-biased dispersal: a review of the theory. Biol. Rev. Camb. Philos. Soc. 94, 721-736.

19. De Lisle, S. P. Understanding the evolution of ecological sex differences: integrating character displacement and the Darwin-Bateman paradigm. Evol. Lett. 3, 434-447 (2019).

20. Wright, S. Size of population and breeding structure in relation to evolution. Science 87, 430-431 (1938).

21. Caballero, A. On the effective size of populations with separate sexes, with particular reference to sex-linked genes. Genetics 139, 1007-1011 (1995).

22. Laporte, V. \& Charlesworth, B. Effective population size and population subdivision in demographically structured populations. Genetics 162, 501-519 (2002).

23. Eberhart-Phillips, L. J. et al. Sex-specific early survival drives adult sex ratio bias in snowy plovers and impacts mating system and population growth. Proc. Natl. Acad. Sci. U. S. A. 114, E5474-E5481 (2017).

24. Regan, C. E., Medill, S. A., Poissant, J., et al. Causes and consequences of an unusually male-biased adult sex ratio in an unmanaged feral horse population. Journal of Animal (2020).

25. Charlesworth, B. The effect of life-history and mode of inheritance on neutral genetic variability. Genet. Res. 77, 153-166 (2001). 
26. Wilson Sayres, M. A. Genetic diversity on the sex chromosomes. Genome Biol. Evol. 10, 1064-1078 (2018).

27. Lind, J. M. et al. Elevated male European and female African contributions to the genomes of African American individuals. Hum. Genet. 120, 713-722 (2007).

28. Hedrick, P. W. Sex: differences in mutation, recombination, selection, gene flow, and genetic drift. Evolution 61, 2750-2771 (2007).

29. Gardner, A. Total reproductive value of juvenile females is twice that of juvenile males under X-linkage and haplodiploidy. J. Theor. Biol. 359, 236-237 (2014).

30. Hammer, M. F., Mendez, F. L., Cox, M. P., Woerner, A. E. \& Wall, J. D. Sex-biased evolutionary forces shape genomic patterns of human diversity. PLoS Genet. 4, e1000202 (2008).

31. Ellegren, $\mathrm{H}$. The different levels of genetic diversity in sex chromosomes and autosomes. Trends Genet. 25, 278-284 (2009).

32. Webster, T. H. \& Wilson Sayres, M. A. Genomic signatures of sex-biased demography: progress and prospects. Curr. Opin. Genet. Dev. 41, 62-71 (2016).

33. Goldberg, A. \& Rosenberg, N. A. Beyond 2/3 and 1/3: the complex signatures of sex-biased admixture on the X chromosome. Genetics 201, 263-279 (2015).

34. Kokko, H. \& Jennions, M. D. The relationship between sexual selection and sexual conflict. Cold Spring Harb. Perspect. Biol. 6, a017517.

35. Corl, A. \& Ellegren, H. The genomic signature of sexual selection in the genetic diversity of the sex chromosomes and autosomes. Evolution 66, 2138-2149 (2012).

36. Oyler-McCance, S. J., Cornman, R. S., Jones, K. L. \& Fike, J. A. Z chromosome divergence, polymorphism and relative effective population size in a genus of lekking birds. Heredity 115, 452-459 (2015).

37. Irwin, D. E. Sex chromosomes and speciation in birds and other ZW systems. Mol. Ecol. 27, 3831-3851 (2018).

38. Keinan, A., Mullikin, J. C., Patterson, N. \& Reich, D. Accelerated genetic drift on chromosome X during the human dispersal out of Africa. Nat. Genet. 41, 66-70 (2009).

39. Emery, L. S., Felsenstein, J. \& Akey, J. M. Estimators of the human effective sex ratio detect sex biases on different timescales. Am. J. Hum. Genet. 87, 848-856 (2010).

40. Ryman, N., Laikre, L. \& Hössjer, O. Do estimates of contemporary effective population size tell us what we want to know? Mol. Ecol. 28, 1904-1918 (2019).

41. Roberts, D. F. Genetic effects of population size reduction. Nature 220, 1084-1088 (1968).

42. Roberts, D. F. \& Bear, J. C. Measures of genetic change in an evolving population. Hum. Biol. 52, 773-786 (1980).

43. MacCluer, J. W., VandeBerg, J. L., Read, B. \& Ryder, O. A. Pedigree analysis by computer simulation. Zoo Biol. 5, 147-160 (1986).

44. Manatrinon, S., Egger-Danner, C. \& Baumung, R. Estimating lethal allele frequencies in complex pedigrees via gene dropping approach using the example of Brown Swiss cattle. Arch. Anim. Breed. 52, 230-242 (2009).

45. Chong, J. X., Ouwenga, R., Anderson, R. L., Waggoner, D. J. \& Ober, C. A population-based study of autosomalrecessive disease-causing mutations in a founder population. Am. J. Hum. Genet. 91, 608-620 (2012).

46. Gratten, J., Pilkington, J. G., Brown, E. A., Clutton-Brock, T. H., Pemberton, J. M. \& Slate, J. Selection and microevolution of coat pattern are cryptic in a wild population of sheep. Mol. Ecol. 21, 2977-2990 (2012).

47. Johnston, S. E., Gratten, J., Berenos, C., Pilkington, J. G., Clutton-Brock, T. H., Pemberton, J. M. \& Slate, J. Life history trade-offs at a single locus maintain sexually selected genetic variation. Nature 502, 93-95 (2013).

48. Kukekova, A. V. et al. Red fox genome assembly identifies genomic regions associated with tame and aggressive behaviours. Nat Ecol Evol 2, 1479-1491 (2018).

49. Fitzpatrick, J. W. \& Bowman, R. Florida scrub-jays: oversized territories and group defense in a fire-maintained habitat. Cooperative breeding in vertebrates: studies of ecology (2016).

50. Coulon, A., Fitzpatrick, J. W., Bowman, R. \& Lovette, I. J. Effects of habitat fragmentation on effective dispersal of Florida Scrub-Jays. Conserv. Biol. 24, 1080-1088 (2010).

51. Aguillon, S. M., Fitzpatrick, J. W., Bowman, R., Schoech, S. J., Clark, A. G., Coop, G. \& Chen, N. Deconstructing isolation-by-distance: The genomic consequences of limited dispersal. PLoS Genet. 13, e1006911 (2017). 
bioRxiv preprint doi: https://doi.org/10.1101/2021.10.28.466320; this version posted October $29,2021$. The copyright holder for this preprint (which was not certified by peer review) is the author/funder, who has granted bioRxiv a license to display the preprint in perpetuity. It is made available under aCC-BY-NC 4.0 International license.

52. Suh, Y. H., Pesendorfer, M. B., Tringali, A., Bowman, R. \& Fitzpatrick, J. W. Investigating social and environmental predictors of natal dispersal in a cooperative breeding bird. Behav. Ecol. 31, 692-701 (2020).

53. Townsend, A. K., Bowman, R., Fitzpatrick, J. W., Dent, M. \& Lovette, I. J. Genetic monogamy across variable demographic landscapes in cooperatively breeding Florida scrub-jays. Behav. Ecol. 22, 464-470 (2011).

54. Chen, N., Cosgrove, E. J., Bowman, R., Fitzpatrick, J. W. \& Clark, A. G. Genomic consequences of population decline in the endangered Florida Scrub-Jay. Curr. Biol. 26, 2974-2979 (2016).

55. Schoech, S. J., Mumme, R. L. \& Moore, M. C. Reproductive endocrinology and mechanisms of breeding inhibition in cooperatively breeding Florida Scrub Jays (Aphelocoma coerulescens). Condor 93, 354-364 (1991).

56. Mumme, R. Do helpers increase reproductive success? Behav. Ecol. Sociobiol. 31 (1992).

57. Fridolfsson, A.-K. \& Ellegren, H. A simple and universal method for molecular sexing of non-ratite birds. J. Avian Biol. 30, 116-121 (1999).

58. Purcell, S. et al. PLINK: a tool set for whole-genome association and population-based linkage analyses. Am. J. Hum. Genet. 81, 559-575 (2007).

59. R Core Team. R: A Language and Environment for Statistical Computing R Foundation for Statistical Computing (Vienna, Austria, 2019). https://www.R-project.org/.

60. Wickham, H. The Split-Apply-Combine Strategy for Data Analysis. Journal of Statistical Software 40, 1-29 (2011).

61. Wickham, H., François, R., Henry, L. \& Müller, K. dplyr: A Grammar of Data Manipulation R package version 1.0.4 (2021). https://CRAN.R-project.org/package=dplyr.

62. Wickham, H. ggplot2: Elegant Graphics for Data Analysis ISBN: 978-3-319-24277-4. https://ggplot2 . tidyverse.org (Springer-Verlag New York, 2016).

63. Wilke, C. O. cowplot: Streamlined Plot Theme and Plot Annotations for 'ggplot2' R package version 0.9.2 (2017). https://CRAN. R-project.org/package=cowplot.

64. Therneau, T. M. \& Sinnwell, J. kinship2: Pedigree Functions R package version 1.6.4 (2015). https ://CRAN. Rproject.org/package=kinship2.

65. Microsoft \& Weston, S. foreach: Provides Foreach Looping Construct for R R package version 1.4.4 (2017). https: //CRAN.R-project.org/package=foreach.

66. Microsoft \& Weston, S. doParallel: Foreach Parallel Adaptor for the 'parallel' Package R package version 1.0.14 (2018). https://CRAN.R-project.org/package=doParallel.

67. Quinn, J. S., Woolfenden, G. E., Fitzpatrick, J. W. \& White, B. N. Multi-locus DNA fingerprinting supports genetic monogamy in Florida scrub-jays. Behav. Ecol. Sociobiol. 45, 1-10 (1999).

68. Reid, J. M., Nietlisbach, P., Wolak, M. E., Keller, L. F. \& Arcese, P. Individuals' expected genetic contributions to future generations, reproductive value, and short-term metrics of fitness in free-living song sparrows (Melospiza melodia). Evol. Lett. 3, 271-285 (2019).

69. Hunter, D. C., Pemberton, J. M., Pilkington, J. G. \& Morrissey, M. B. Pedigree-based estimation of reproductive value. J. Hered. 110, 433-444 (2019).

70. Grafen, A. A theory of Fisher's reproductive value. J. Math. Biol. 53, 15-60 (2006).

71. Lanciani, C. A. A simple equation for presenting reproductive value to introductory biology and ecology classes. Bulletin of the Ecological Society of America 79, 192-193 (1998).

72. Robertson, A. Inbreeding in artificial selection programmes. Genet. Res. 2, 189-194 (1961).

73. Buffalo, V., Mount, S. M. \& Coop, G. A genealogical look at shared ancestry on the X chromosome. Genetics 204, 57-75 (2016).

74. Grafen, A. Total reproductive values for females and for males in sexual diploids are not equal. J. Theor. Biol. 359, 233-235 (2014).

75. Ruzicka, F. et al. The search for sexually antagonistic genes: Practical insights from studies of local adaptation and statistical genomics. Evolution Letters (2020).

76. Otto, S. P. et al. About PAR: the distinct evolutionary dynamics of the pseudoautosomal region. Trends Genet. 27, 358-367 (2011). 